\title{
Nanostrip-Induced High Tunability Multipolar Fano Resonances in a Au Ring-Strip Nanosystem
}

\author{
Zao $\mathrm{Yi}^{1,2}$, Xin Li ${ }^{1,2}$, Xibin $\mathrm{Xu}^{3}$, Xifang Chen ${ }^{1,2}$, Xin Ye ${ }^{3, *}$, Yong $\mathrm{Yi}^{1,2}$, Tao Duan ${ }^{1,2}$, \\ Yongjian Tang ${ }^{1,2}$, Jiangwei Liu ${ }^{4, *}$ and Yougen $\mathrm{Yi}^{5, *}$ \\ 1 Joint Laboratory for Extreme Conditions Matter Properties, Southwest University of Science and Technology, \\ Mianyang 621010, China; yizaomy@swust.edu.cn (Z.Y.); lixin1010106@yeah.net (X.L.); \\ chenxifang1988@yeah.net (X.C.); twcsu2013@csu.edu.cn (Y.Y.); myyz1984@csu.edu.cn (T.D.); \\ tangyongjian2000@yeah.net (Y.T.) \\ 2 Sichuan Civil-Military Integration Institute, Mianyang 621010, China \\ 3 Research Center of Laser Fusion, China Academy of Engineering Physics, Mianyang 621900, China; \\ dodolong@csu.edu.cn \\ 4 School of Energy Science and Engineering, Central South University, Changsha 410083, China \\ 5 College of Physics and Electronics, Central South University, Changsha 410083, China \\ * Correspondence: yexin@caep.cn (X.Y.); jiangweiliu@csu.edu.cn (J.L.); yougenyi@csu.edu.cn (Y.Y.); \\ Tel./Fax: +86-816-2780-827 (X.Y.); +86-731-8883-6457 (J.L. \& Y.Y.)
}

Received: 26 June 2018; Accepted: 23 July 2018; Published: 25 July 2018

\begin{abstract}
Surface plasmon resonances of a Au ring-strip nanosystem with tunable multipolar Fano resonances have been investigated based on the finite-difference time-domain (FDTD) method. Abundant plasmon properties of a Au ring-strip nanosystem can be obtained on the basis of the unique electronic properties of different geometry parameters. In our research models, these multipolar Fano resonances are induced and can be tuned independently by changing the geometry parameters of the $\mathrm{Au}$ ring-strip nanosystem. Complex electric field distributions excited by the Au ring-strip nanosystem provide possibility to form dark plasmonic modes. Multipolar Fano resonances display strong light extinction in the Au ring-strip nanosystem, which can offer a new approach for an optical tunable filter, optical switching, and advanced biosensing.
\end{abstract}

Keywords: Au ring-strip nanosystem; multipolar Fano resonances; surface plasmon resonances; FDTD method

\section{Introduction}

In recent years, due to the ability to control light at visible and near-infrared wavelengths, and thereby induce very large electric field enhancement near the metallic micro/nanostructure surfaces, localized surface plasmon resonance (LSPR) of the metallic micro/nanostructure has been studied intensively [1-5]. LSPR has intriguing applications such as subwavelength waveguiding [6], biosensing [7], surface-enhanced Raman spectroscopy (SERS) [8], photovoltaic devices [9], and optical traps [10]. Recently, Fano resonances which arise from the interaction between bright and dark plasmonic modes are an interesting result of electromagnetic coupling in metallic micro/nanostructure [11,12]. Originating from quantum mechanics, Fano resonances are a general physical phenomenon found in systems comprising a coupling between a broad continuum of energy levels and discrete states. Due to their sensitive characteristic, Fano resonances have attracted significant attentions and have been used for biochemical sensing [13], second harmonic generation [14], SERS [15], nonlinear optics [16], and so on.

As Fano resonances arise from the coupling of micro/nanostructures, they are sensitive to the changes in geometry parameters of the micro/nanostructures: small perturbations can induce dramatic resonance or lineshape shifts. Plasmonic Fano resonance has been reported in a large number of metallic 
micro/nanostructure systems, such as nanorod complexes [17], core-shell nanoparticles [18], ring-disk nanostructures [19], oligomer clusters [20], nanoholes [21], and so on. The intensity and position of Fano resonances are mainly tuned by coupling with bright and dark plasmonic modes, which can be determined by the parameters of the nanostructure [22-24]. The effective achievement of the dark plasmonic modes in nanostructure is still an open question, because the dark plasmonic modes could not effectively couple with the electric field of the incident light once the wavelength of the excitation light is much greater than the size of the nanostructure. Dark plasmonic modes can be induced in large nanostructures because of the phase retardation effect. However, those dark plasmonic modes were usually excited by an electric field with grazing light, which hampers practical applications because most of these applications depend on the excitation of resonances by light at normal incidence [25-27]. Up to now, it is still a very large challenge to design nanostructures exhibiting tunable plasmonic Fano resonances at visible and near-infrared wavelengthss for their practical applications.

The multiple Fano resonances have been reported in previous literatures [28,29], but in the normal case, the multiple dark modes are simultaneously excited. Inspired by the experimental work of Lee et al. [30], in our research work, we designed Au ring-strip nanstructures to study the plasmonic multipolar Fano resonances. Compared with the nanostructure with a nanorod dimer and nanoring proposed by Lee et al. the multiple Fano resonances of our designed nanostructure are easier to be adjusted to the visible and near infrared by regulating the structural parameters. A variety of geometric parameters of Au ring-strip nanosystem were designed to regulate and control the plasmonic Fano resonances. Unique electronic and optical properties will induce new plasmonic Fano resonance properties in the Au ring-strip nanosystem. We hope the Au ring-strip nanosystem will provide new applications in surface-enhanced spectroscopy and optical switches.

\section{Theoretical Methods}

The plasmonic Fano resonances and near-field intensity distributions of the Au ring-strip nanosystem were simulated based on the finite-difference time-domain (FDTD) method. As shown in Figure 1A, the incident light wave polarized along the x-direction was illuminated from the $\mathrm{SiO}_{2}$ substrate, and it was perpendicular to the Au ring-strip nanosystem. The perfectly-matched layer absorbing boundaries were chosen as simulated boundary conditions. The simulation region is $0.14 \times 0.14 \times 0.14 \mu \mathrm{m}$, and the cell size is $0.5 \times 0.5 \times 0.5 \mathrm{~nm}$. In this study, the dielectric functions of the $\mathrm{Au}$ ring-strip nanosystem were described using the experimental data of Johnson and Christy [31]. The refractive index of surrounding medium was set to be 1.0. The dielectric functions of the $\mathrm{SiO}_{2}$ were described by Palik [32]. Figure 1A,B display the detailed schematic of the simulated nanostructures. The structural parameters of the Au nanoring (outer radius $\left(\mathrm{R}_{2}\right)$, inner radius $\left(\mathrm{R}_{1}\right)$, and thickness $\left(\mathrm{H}_{1}\right)$ ) and nanostrip (length $(\mathrm{L})$, width $(\mathrm{W})$ and thickness $\left(\mathrm{H}_{2}\right)$ ) are shown in Figure 1A. In our research, we fixed the same thickness of $\mathrm{H}_{1}=\mathrm{H}_{2}=10 \mathrm{~nm}$.

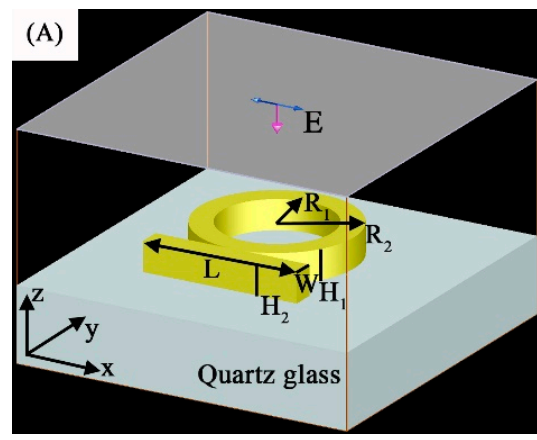

(B)
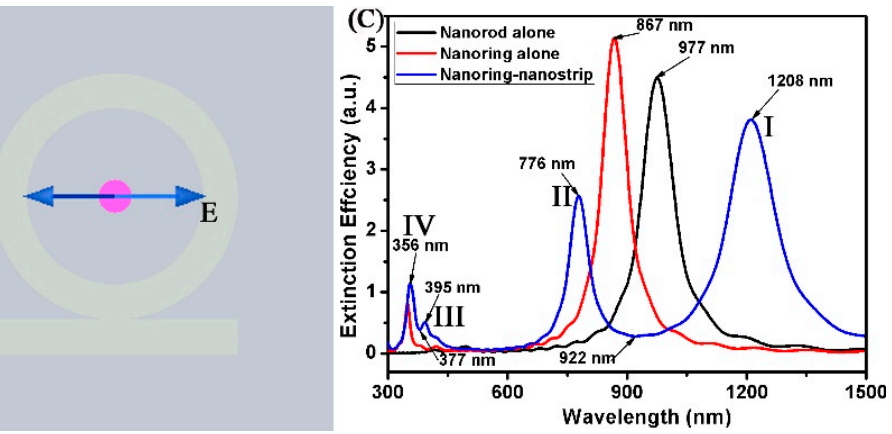

Figure 1. (A,B) Schematic illustration of the Au ring-strip nanosystem used in the simulation. (C) Extinction spectra of the Au nanostrip alone (black curve), the Au nanoring alone (red curve), and the Au ring-strip nanosystem (blue curve). 


\section{Results and Discussion}

Figure $1 \mathrm{C}$ shows the extinction spectra of different models. In this case, for the Au nanoring, $R_{2}$ and $R_{1}$ are $30 \mathrm{~nm}$ and $20 \mathrm{~nm}$, respectively. For the Au nanostrip, $L$ is $60 \mathrm{~nm}$ and $W$ is $10 \mathrm{~nm}$. The polarization direction of the plane wave is parallel to the $\mathrm{x}$-axis. As depicted in Figure $1 \mathrm{C}$ (the black curve), for the incident light wave polarized along the long axis of the Au nanostrip, the extinction spectra has a longitudinal dipole resonance peak at $977 \mathrm{~nm}$ [33]. As shown by the red curve, for a single Au nanoring, the spectrum displays the transverse dipole bonding mode at $867 \mathrm{~nm}$, which is a result of the symmetric coupling between the internal and external surfaces of the Au nanoring dipolar modes [34]. As shown by the blue curve, for Au ring-strip nanosystem, the extinction spectra shows very different absorption peaks. As shown in Figure 1A,B, the Au nanoring and nanostrip are combined into a symmetric ring-strip composite nanostructure. The extinction spectra of Au ring-strip nanosystem displays four optical resonances. In addition to a transverse dipole bonding mode I (the bright plasmonic mode) at $1208 \mathrm{~nm}$, higher resonance modes of II (the dark plasmonic mode), III (the dark plasmonic mode), and IV (the bright plasmonic mode) are observed at $776 \mathrm{~nm}$, $395 \mathrm{~nm}$, and $356 \mathrm{~nm}$, respectively. This may be because polarized light can excite both symmetric and anti-symmetric modes simultaneously for the composite structure. We define the quality factor (Q) as the resonant wavelength over the width of the resonance. The Q factor of I, II, III, and IV modes are 8.9, $14.4,20.4$, and 38.3, respectively. As shown by the blue curve, two apparent minimum resonance peaks at the resonance wavelength of $922 \mathrm{~nm}$ and $377 \mathrm{~nm}$ were found, where the dark plasmonic mode and the bright plasmonic mode are spectrally overlapped. The excellent dark plasmonic modes excited in the $\mathrm{Au}$ ring-strip nanosystem can make them good choices to interact well with the bright plasmonic modes, where the plasmonic Fano resonances can be easily tuned.

Figure 2 displays the field distributions of Au ring-strip nanosystem surfaces at four strong absorption peaks located at 1208, 776, 395, and $356 \mathrm{~nm}$, and two minimum absorption peaks located at 922 and $377 \mathrm{~nm}$. The field distributions in Figure 2A display local field of the resonance peak at $1208 \mathrm{~nm}$. Here, the dipole-dominant field distributions are along the x-polarization. The field distributions are around the outer surface of the Au nanoring and two ends of the nanostrip. As shown in Figure 2B, the strong field distributions mainly appear at both ends of the nanostrip. The weak charge distributions on the Au nanoring exhibit four nodes, which implies quadrupole plasmon modes there. Therefore, the resonance at $776 \mathrm{~nm}$ is the dark plasmonic resonance mode. As shown in Figure 2C, the electric field distributions are similar to that of Figure 2B, indicating that they are also the dark field mode. As shown in Figure 2D, the strong electric field distributions are concentrated at the junction between the nanoring and nanostrip. According to an analysis of the absorption peaks at 1208, 776, 395, and $356 \mathrm{~nm}$, minimum resonances at 922, and $377 \mathrm{~nm}$ provide the possibility to produce multipolar Fano resonances. As shown in Figure 2E, the field distributions at $922 \mathrm{~nm}$ present a very complex scene. As we know, plasmon oscillations at Fano resonance can be dispersed to localized regions. Therefore, bright plasmonic mode at $1208 \mathrm{~nm}$ and dark plasmonic mode at $776 \mathrm{~nm}$ can produce the Fano resonance at $922 \mathrm{~nm}$. As shown in Figure 2F, the multipolar field distributions mainly appear around the inner surface of nanoring. Due to the coupling of bright and dark plasmonic modes, the complex field distributions were generated.

As we know, the plasmon characteristics of Au nanorods are mainly affected by the longitudinal dipole modes $[35,36]$. In our study, we can also predict that the geometry parameters of Au nanostrips will also affect the plasmon characteristics of the Au ring-strip nanosystem. Figure 3 displays the extinction spectra of the ring-strip nanosystem with different $\mathrm{L}$ of Au nanostrip. In this case, the $\mathrm{R}_{2}$ and $R_{1}$ of the Au nanoring are fixed at $30 \mathrm{~nm}$ and $20 \mathrm{~nm}$, respectively. The $W$ of the Au nanostrip is fixed at $10 \mathrm{~nm}$. The $\mathrm{L}$ of the Au nanostrip varies from 10 to $180 \mathrm{~nm}$. Due to the coupling of the nanoring and nanostrip, these nanostructures display four well-defined peaks at I, II, III, and IV. However, with the increase of the $\mathrm{L}$ of Au nanostrip, the extinction peaks of different modes show different changes. When the L changes from 10 to $180 \mathrm{~nm}$, the dipole resonances of mode I red shift from 913 to $1660 \mathrm{~nm}$. Additionally, the relative intensity ratio of dipole resonances reduces from 6.8 
to 2.8. For Au nanorods, the dipole resonances red shift and relative intensity ratio of dipole resonances decreases with increasing aspect ratio $[35,36]$. In the Au ring-strip nanosystem, the charge assigned to the $\mathrm{Au}$ nanoring will increase with the increase of the $\mathrm{L}$ of nanostrip. This explains why the relative intensity ratio of resonances of mode II increases with the increase of the L of nanostrip, which increases from 1.2 to 3.3. When the $\mathrm{L}$ of the Au nanostrip varies from 10 to $180 \mathrm{~nm}$, the resonances of mode II have red shift and the extinction efficiency has increased. The resonances of mode II red shift from $748 \mathrm{~nm}(\mathrm{~L}=10 \mathrm{~nm})$ to $787 \mathrm{~nm}(\mathrm{~L}=80 \mathrm{~nm})$. At the same time, the extinction efficiency increases from 1.2 to 2.7. When the $\mathrm{L}$ of the Au nanostrip varies from 80 to $180 \mathrm{~nm}$, the resonances of mode III red shift from 395 to $398 \mathrm{~nm}$, and the resonances of mode IV blue shift from 353 to $346 \mathrm{~nm}$. The corresponding extinction spectra of the Au ring-strip nanosystem exhibit Fano resonances, where the Fano resonances red shift from 829 to $1115 \mathrm{~nm}$ with the L of nanostrip increasing from 20 to $180 \mathrm{~nm}$. The spectrum modulations from visible and near infrared light are important for sensing.

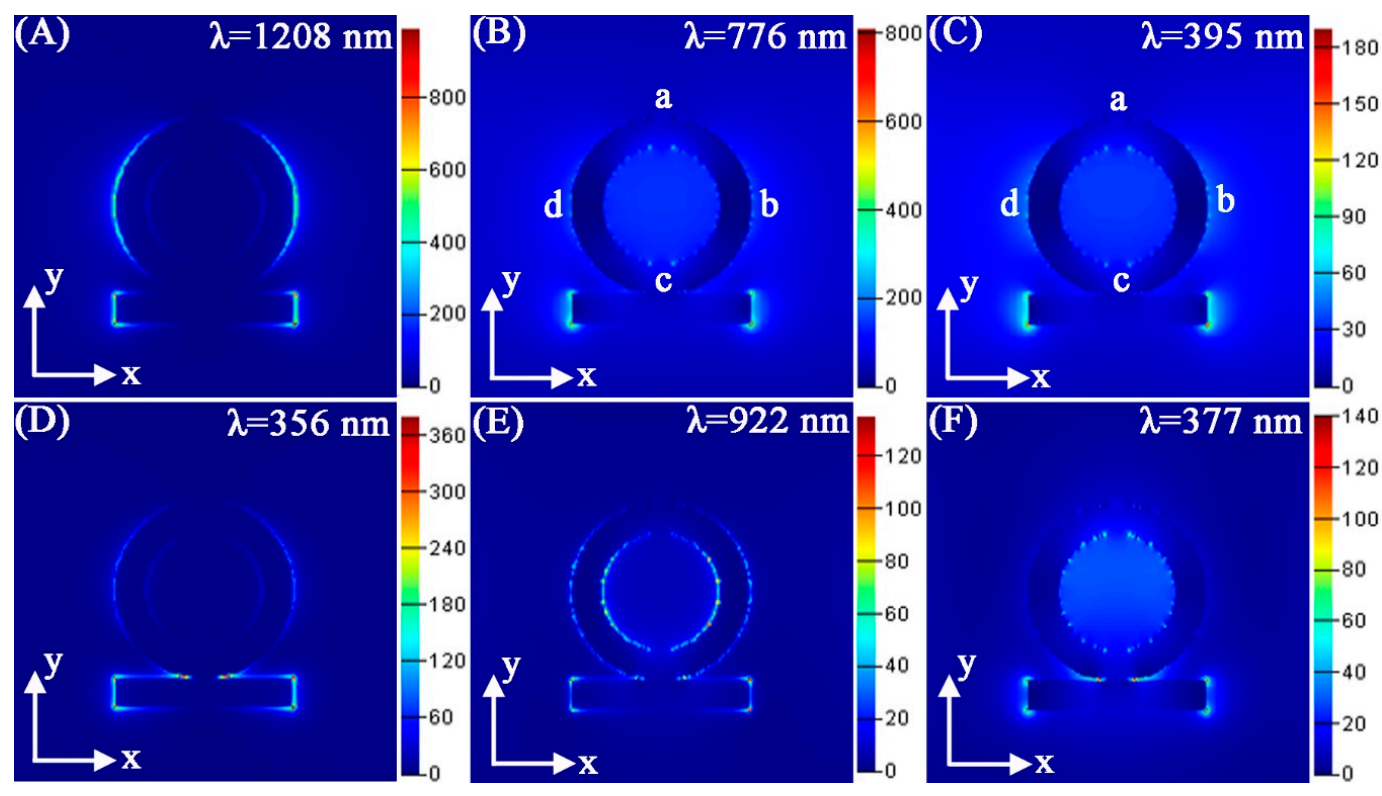

Figure 2. (A-F) Calculated electric field $\left|\mathrm{E}_{2}\right| /\left|\mathrm{E}_{0}{ }^{2}\right|$ of the Au ring-strip nanosystem at different resonance wavelengths. The structure parameters are as follows: $W=10 \mathrm{~nm}, R_{1}=20 \mathrm{~nm} ; R_{2}=30 \mathrm{~nm}$; $\mathrm{L}=60 \mathrm{~nm}$. The color bars in the logarithmic scale for electric enhancement are shown (the units are $\left.(\mathrm{V} / \mathrm{m})^{2}\right)$.
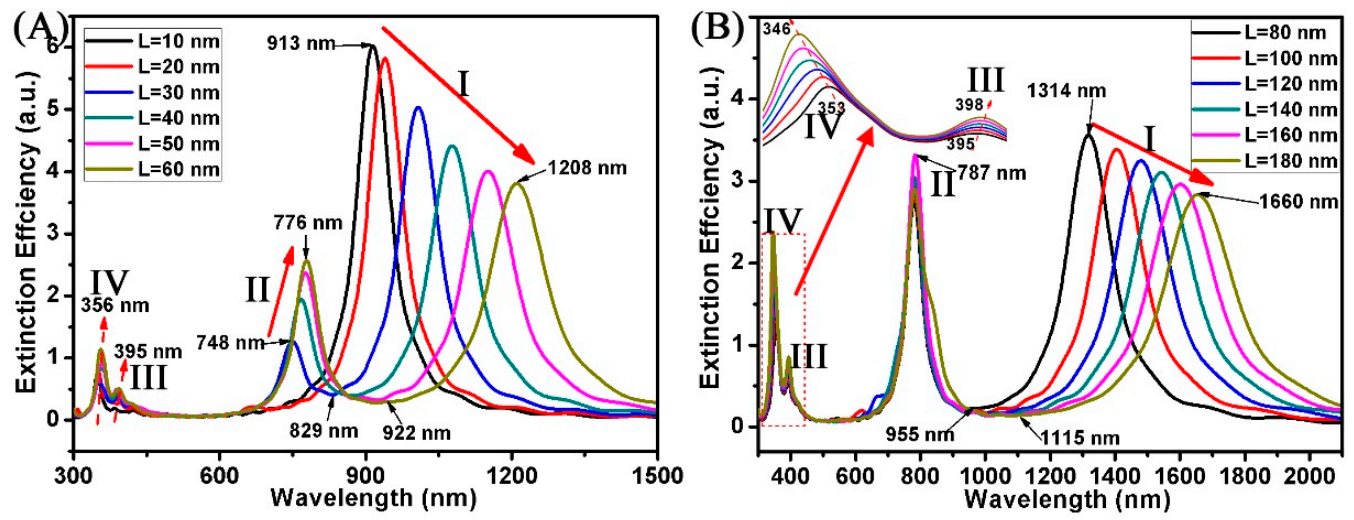

Figure 3. (A,B) Extinction spectra of the Au ring-strip nanosystem with different $A u$ nanostrip length (L). The radius of the Au nanoring are unchanged $\left(R_{2}=30 \mathrm{~nm} ; R_{1}=20 \mathrm{~nm}\right)$. W of the Au nanostrip remains unchanged $(\mathrm{W}=10 \mathrm{~nm})$. L of the Au nanostrip increases from 10 to $180 \mathrm{~nm}$. 
Figure 4 shows the extinction spectra of the Au ring-strip nanosystem with different $\mathrm{W}$ of Au nanostrip. In this case, the $R_{2}$ and $R_{1}$ of the Au nanoring are fixed at $20 \mathrm{~nm}$, and $30 \mathrm{~nm}$, respectively. The $\mathrm{L}$ of the Au nanostrip is fixed at $60 \mathrm{~nm}$. The $\mathrm{W}$ of the Au nanostrip varies from 2 to $60 \mathrm{~nm}$. Here, the $\mathrm{W}$ for simulation can be classified into two types of cases, one with the $\mathrm{W}$ of nanostrip greater than the fixed thickness of the nanoring $\left(t, t=R_{2}-R_{1}=10 \mathrm{~nm}\right)$, and the other smaller than it. When the $\mathrm{W}$ varies from 2 to $10 \mathrm{~nm}(\mathrm{~W}<\mathrm{t})$, as shown in Figure $4 \mathrm{~A}$, the resonances of mode I blue shift from 1296 to $1208 \mathrm{~nm}$. The relative intensity ratio of resonances increases from 1.7 to 3.8. The reason is that the ratio of the surface atoms to total atoms in the nanostructure varies. When all other parameters are fixed, the ratio increases when the $\mathrm{W}$ of the Au nanostrip increases. As the $\mathrm{W}$ of the nanostrip varies from 2 to $10 \mathrm{~nm}$, the resonances of mode II have blue shift and the extinction efficiency has decreased. The resonances of mode II blue shift from $819 \mathrm{~nm}(\mathrm{~W}=2 \mathrm{~nm})$ to $776 \mathrm{~nm}(\mathrm{~W}=10 \mathrm{~nm})$. At the same time, the extinction efficiency decreases from 3.8 to 2.6. When the $W$ of the nanostrip increases, the electrons transferred to the Au nanoring will decrease. Therefore, the absorption peaks (mode II) blue shift and the intensity decreases. The Fano resonances blue shift from 1026 to $922 \mathrm{~nm}$ with the $\mathrm{W}$ of nanostrip increasing from 2 to $10 \mathrm{~nm}$. As shown in Figure 4B, when the $W$ of nanostrips is larger than $10 \mathrm{~nm}$, variation trend of absorption peaks has distinct differences, and some new absorption peaks appeared in these curves. When the $\mathrm{W}$ of the nanostrip is $10 \mathrm{~nm}$, the resonance of mode I has the largest blue shift $(1208 \mathrm{~nm})$ in all the curves. When the $W$ varies from 20 to $60 \mathrm{~nm}(W>t)$, as shown in Figure 4B, the resonances of mode I have red shift and the extinction efficiency has increased. The resonances of mode I red shift from $1229 \mathrm{~nm}(\mathrm{~W}=20 \mathrm{~nm})$ to $1357 \mathrm{~nm}(\mathrm{~W}=60 \mathrm{~nm})$. At the same time, the extinction efficiency increases from 4.4 to 8.3. However, the resonances of mode II are maintained at $770 \mathrm{~nm}$. This indicates that the nanostrip does not affect the resonances of mode II when the $\mathrm{W}$ of the nanostrip is greater than $10 \mathrm{~nm}$. Since the peak shift of mode I and mode II is not significant, the Fano resonances are maintained at $922 \mathrm{~nm}$. When the $\mathrm{W}$ of the nanostrip is greater than the thickness $(t)$ of the nanoring, the Fano resonances are not suitable adjustable. When the $\mathrm{W}$ varies from 40 to $60 \mathrm{~nm}(\mathrm{~W} \geq 2 \mathrm{t})$, as shown in Figure 4B, some new multipolar absorption peaks (V, VI, and VII modes) appeared in these curves.
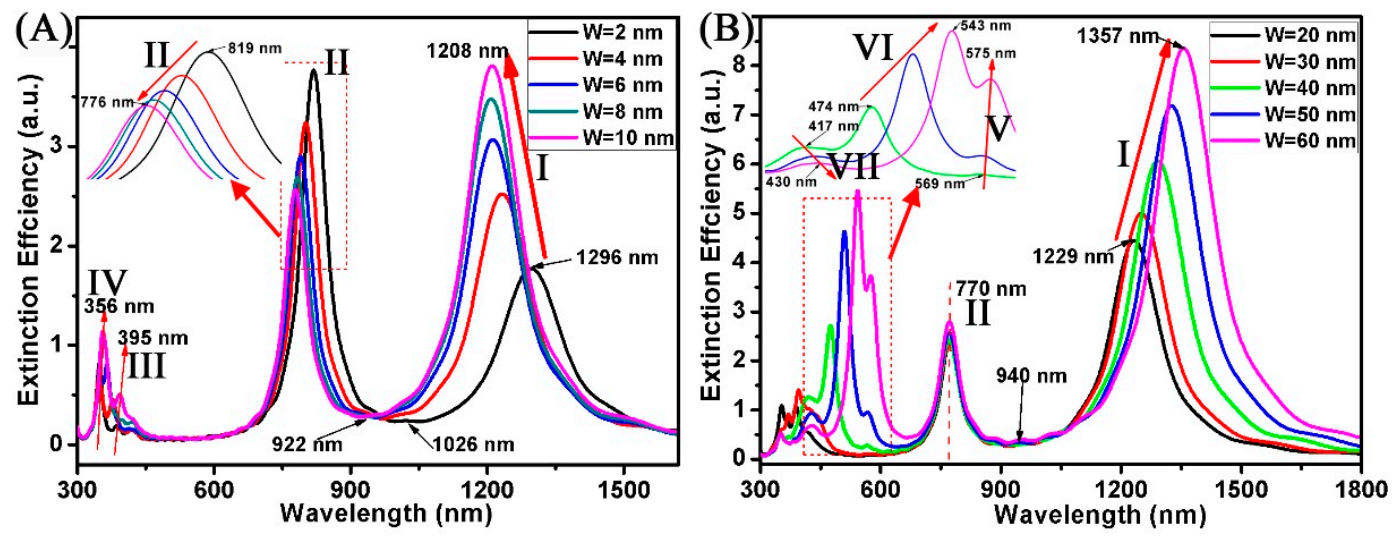

Figure 4. (A,B) Extinction spectra of the Au ring-strip nanosystem with different $W$ of the Au nanostrip. The radius of the Au nanoring are unchanged $\left(R_{2}=30 \mathrm{~nm} ; R_{1}=20 \mathrm{~nm}\right)$. L of the Au nanostrip is unchanged $(\mathrm{L}=60 \mathrm{~nm})$. W of the Au nanostrip increases from 2 to $60 \mathrm{~nm}$.

In our previous studies, the increase of nanoring radius can effectively promote the red shift of the absorption peak of the surface plasmon [37]. Figure 5 displays the extinction spectra of the $\mathrm{Au}$ ring-strip nanosystem with different $R_{1}$ of Au nanoring. In this case, the $R_{2}$ of the Au nanoring is fixed at $30 \mathrm{~nm}$. The $\mathrm{L}$ and $\mathrm{W}$ of the Au nanostrip are fixed at 60 and $10 \mathrm{~nm}$, respectively. The $R_{2}$ of the Au nanoring vary from 0 to $28 \mathrm{~nm}$. When the $R_{1}$ varies from 20 to $28 \mathrm{~nm}$, as shown in Figure $5 \mathrm{~A}$, the resonances of mode I and mode II red shift and the extinction efficiency is decreased, and the shift 
range of Fano resonances is very large, which red shift from 922 to $1450 \mathrm{~nm}$. When the $\mathrm{R}_{1}$ varies from 10 to $20 \mathrm{~nm}$, as shown in Figure 5B, the red shift range of resonances (mode I and mode II) is decreasing, which red shift from 1067 to $1208 \mathrm{~nm}$ and red shift from 611 to $776 \mathrm{~nm}$. For mode II, the extinction efficiency is increased. When the $R_{1}$ is $20 \mathrm{~nm}$, and the thickness $\left(t, t=R_{2}-R_{1}\right)$ of nanoring is $10 \mathrm{~nm}$, the extinction efficiency of the resonances (mode I) has a maximum value (3.8), and the red shift range of Fano resonances decreases, red shifting from 799 to $922 \mathrm{~nm}$. When $\mathrm{R}_{1}$ varies from 0 to $8 \mathrm{~nm}$, as shown in Figure 5C, the resonances of mode I are maintained at $1060 \mathrm{~nm}$. For the resonances of mode II, the red shift range is from 560 to $597 \mathrm{~nm}$. When $R_{1}$ is $6 \mathrm{~nm}$, the extinction efficiency of resonances (mode II) has a maximum value (6.9). Since the shift range of mode I and mode II is very small, the shift range of Fano resonances red shift from 765 to $780 \mathrm{~nm}$ only. Here, when the $\mathrm{R}_{1}$ is less than $8 \mathrm{~nm}$, new additional absorption peaks (mode V) appear at $514 \mathrm{~nm}$, which is a result of dipolar plasmon mode of nanodisk [38]. Here, when the $\mathrm{R}_{1}$ increases to $8 \mathrm{~nm}$, the new Fano resonances at $527 \mathrm{~nm}$ gradually weaken until they disappear. For a single Au nanodisk, as shown by the red curve in Figure 5D, the spectrum shows a dipolar excitation at $583 \mathrm{~nm}$ only.
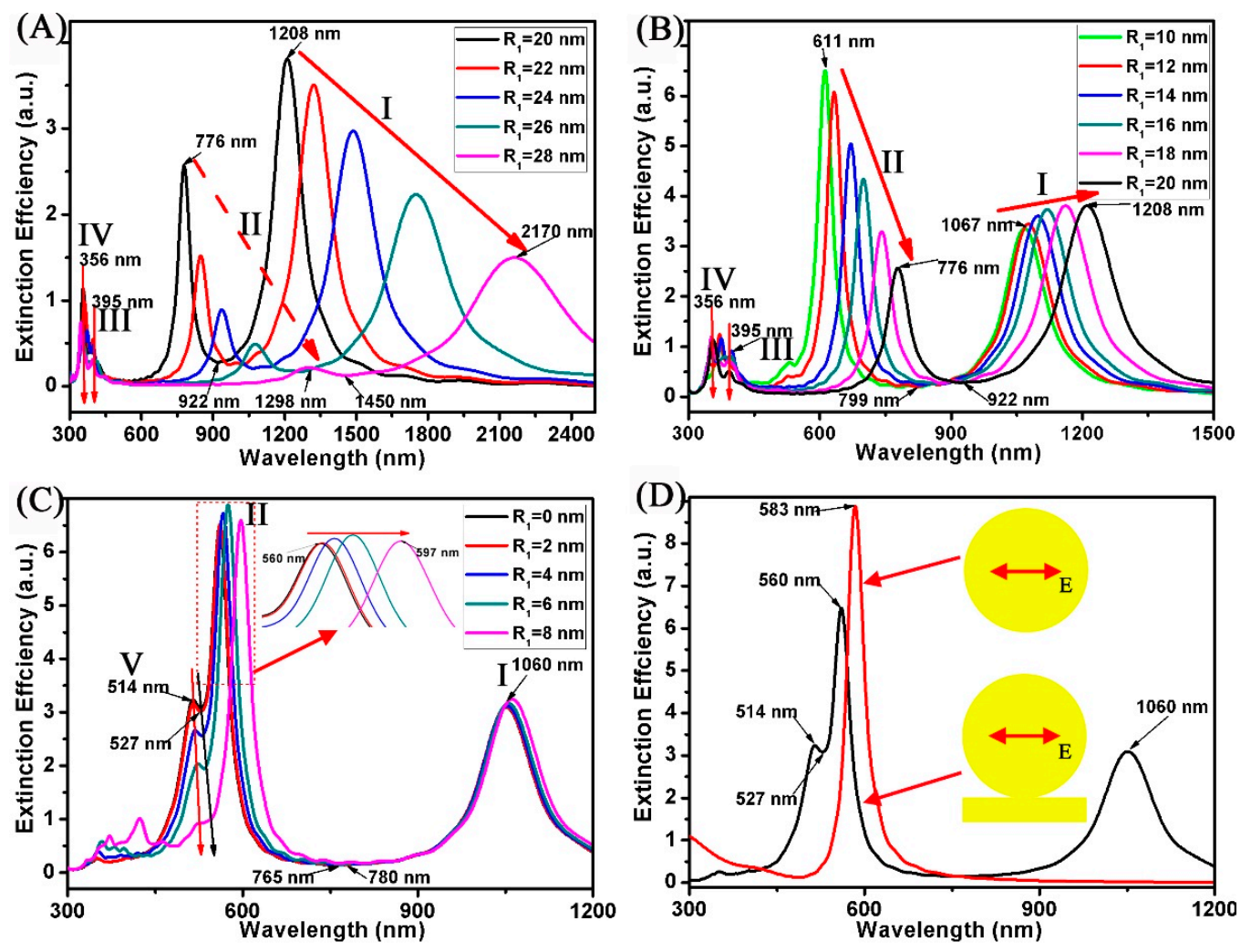

Figure 5. (A-D) Extinction spectra of the Au ring-strip nanosystem with different $R_{1}$ of the Au nanoring. The outer radius of the Au nanoring are unchanged $\left(R_{2}=30 \mathrm{~nm}\right)$. L and $W$ of the Au nanostrip are unchanged $(\mathrm{L}=60 \mathrm{~nm} ; \mathrm{W}=10 \mathrm{~nm})$. $\mathrm{R}_{1}$ of the Au nanoring increases from 0 to $28 \mathrm{~nm}$.

The multipolar Fano resonances characteristics of the proposed nanostructure are also dependent on the polarization direction of the incident light $[39,40]$. Figure 6 displays the calculated extinction spectra of the Au ring-strip nanosystem with different angles $\theta$ ( $\theta$ is the angle between the incident light and x-axis). The structural parameters are fixed ( $\left.W=10 \mathrm{~nm} ; \mathrm{R}_{1}=20 \mathrm{~nm} ; \mathrm{R}_{2}=30 \mathrm{~nm} ; \mathrm{L}=60 \mathrm{~nm}\right)$. The $\theta$ changes from $0^{\circ}$ to $90^{\circ}$. It can be found for Au ring-strip nanosystem with different $\theta$ between $15^{\circ}$ and $75^{\circ}$, five SPR peaks appear in these curves. For instance, at $\theta=45^{\circ}$, five SPR peaks appear at 351 , 392, 576, 776, and $1208 \mathrm{~nm}$, respectively. Here, these SPR resonances are designated as five modes (I, II, III, IV, and V modes). As shown in Figure 6, when the $\theta$ changes from $0^{\circ}$ to $90^{\circ}$, the extinction efficiency of resonances (mode I) gradually decreases to zero. However, for the absorption peaks at $776 \mathrm{~nm}$ 
(mode II), the extinction efficiency increases from 2.6 to 7.4 . When $\theta$ changes from $0^{\circ}$ to $75^{\circ}$, the Fano resonances are maintained at $922 \mathrm{~nm}$. However, when $\theta$ is $90^{\circ}$, the Fano resonance at $922 \mathrm{~nm}$ disappears. The reason is that the Fano resonances can be effectively excited by Ex component of incident light because of the strong interaction between the I and II modes. When $\theta$ is $90^{\circ}$, because only the Ey component exists, mode II can be effectively excited while mode I cannot be excited. When $\theta$ varies from $15^{\circ}$ to $90^{\circ}$, new absorption peaks (mode V) appear at $576 \mathrm{~nm}$. The imperfect Fano resonances at $618 \mathrm{~nm}$ are induced because of the interactions among the dipole mode and the quadrupole mode, which are induced by the Ex and Ey components of the incident light. The Fano resonances at $618 \mathrm{~nm}$ gradually weaken until they disappear with the decrease of $\theta$, accompanyied with the process from a strong coupling to a small coupling between the excited dipole mode and the quadrupole mode. When the incident light is perpendicular to the long axis of the Au nanostrip $\left(\theta=0^{\circ}\right)$, as shown by the red curve in Figure 6B, the extinction spectra has a dipole resonance peak at $349 \mathrm{~nm}$ with weak intensity [40].
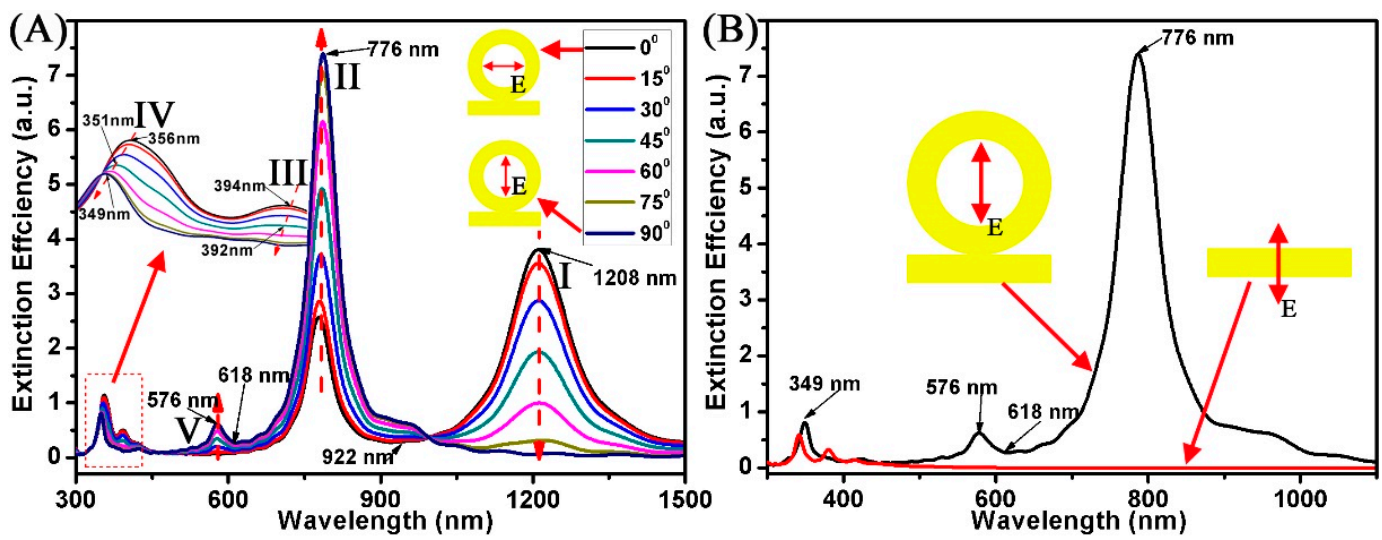

Figure 6. (A,B) Extinction spectra of the Au ring-strip nanosystem with different polarization direction $(\theta)$. Other parameters are unchanged $\left(W=10 \mathrm{~nm} ; \mathrm{R}_{1}=20 \mathrm{~nm} ; \mathrm{R}_{2}=30 \mathrm{~nm} ; \mathrm{L}=60 \mathrm{~nm}\right)$. The $\theta$ increases from $0^{\circ}$ to $90^{\circ}$.

\section{Conclusions}

In summary, the combination of nanostrip in Au ring-strip nanosystems has been proved to be effective in adjusting the surface plasmon frequency. The extinction spectra of Au ring-strip nanosystems display different absorption modes of the plasmon compared with only the nanoring or nanostrip. The surface plasmon interaction at the interface between ring and disk strip can alter the SPR of the Au ring-strip nanosystems greatly. These extinction spectra of Au ring-strip nanosystems with different geometry parameters display the multipolar surface plasmons modes. By regulating geometry parameters, these Fano resonances have wide regulation from visible light to mid-infrared. Furthermore, simulation of Au ring-strip nanosystems with different polarization directions of incident light $(\theta)$ has also been considered in our study. The change of the polarization direction can control the generation and extinction of these Fano resonances. We hope that the Au ring-strip nanosystems can provide a new application for designing a plasmonic Fano switch and multiwavelength surface-enhanced spectroscopy.

Author Contributions: Z.Y., X.Y., and X.L. conceived and designed the simulation; Z.Y. performed the simulation; Z.Y., X.X., X.C., Y.Y. (Yong Yi), T.D., X.Y., Y.T., and Y.Y. (Yougen Yi) analyzed the data; Z.Y., X.Y., J.L., and Y.Y. (Yougen $\mathrm{Yi}$ ) contributed analysis tools; and Z.Y. wrote the paper.

Funding: This research received no external funding.

Acknowledgments: The work is supported by the National Natural Science Foundation of China (No. 51606158; 11604311), the Funded by Longshan academic talent research supporting program of SWUST (No. 18lzx506). 
Conflicts of Interest: The authors declare no conflict of interest.

\section{References}

1. Barnes, W.L.; Dereux, A.; Ebbesen, T.W. Surface plasmon sub-wavelength optics. Nature 2003, 424, 824-830. [CrossRef] [PubMed]

2. Chen, J.J.; Zeng, Y.; Xu, X.B.; Chen, X.F.; Zhou, Z.G.; Shi, P.C.; Yi, Z.; Ye, X.; Xiao, S.Y.; Yi, Y.G. Plasmonic Absorption Enhancement in Elliptical Graphene Arrays. Nanomaterials 2018, 8, 175. [CrossRef] [PubMed]

3. Hu, S.; Yang, H.L.; Han, S.; Huang, X.; Xiao, B.X. Tailoring dual-band electromagnetically induced transparency in planar metamaterials. J. Appl. Phys. 2015, 117, 043107. [CrossRef]

4. Zhukov, A.V.; Bouffanais, R.; Konobeeva, N.N.; Belonenko, M.B. Two-dimensional electroacoustic waves in silicene. Appl. Phys. B 2018, 124, 10. [CrossRef]

5. Liu, L.; Chen, J.J.; Zhou, Z.G.; Yi, Z.; Ye, X. Tunable absorption enhancement in electric split-ring resonators-shaped graphene array. Mater. Res. Express 2018, 5, 045802. [CrossRef]

6. Zhang, S.P.; Wei, H.; Bao, K.; Hakanson, U.; Halas, N.J.; Nordlander, P.; Xu, H.X. Chiral surface plasmon polaritons on metallic nanowires. Phys. Rev. Lett. 2011, 107, 096801. [CrossRef] [PubMed]

7. Anker, J.N.; Hall, W.P.; Lyandres, O.; Shan, N.C.; Zhao, J.; Van-Duyne, R.P. Biosensing with plasmonic nanosensors. Nat. Mater. 2008, 7, 442-453. [CrossRef] [PubMed]

8. Tan, T.X.; Tian, C.G.; Ren, Z.Y.; Yang, J.; Chen, Y.J.; Sun, L.; Li, Z.T.; Wu, A.P.; Yin, J.; Fu, H.G. LSPR-dependent SERS performance of silver nanoplates with highly stable and broad tunable LSPRs prepared through an improved seed-mediated strategy. Phys. Chem. Chem. Phys. 2013, 15, 21034-21042. [CrossRef] [PubMed]

9. Liu, J.Q.; Wang, L.L.; He, M.D.; Huang, W.Q.; Wang, D.; Zou, B.S.; Wen, S.C. A wide bandgap plasmonic Bragg reflector. Opt. Express 2008, 16, 4888-4894. [CrossRef] [PubMed]

10. Liu, W.; Zhang, J.; Zhu, Z.; Yuan, X.; Qin, S. Electrically Tunable Absorption Enhancement with Spectral and Polarization Selectivity through Graphene Plasmonic Light Trapping. Nanomaterials 2016, 6, 155. [CrossRef] [PubMed]

11. Cen, C.L.; Chen, J.J.; Liang, C.P.; Huang, J.; Chen, X.F.; Tang, Y.J.; Yi, Z.; Xu, X.B.; Yi, Y.G.; Xiao, S.Y. Plasmonic absorption characteristics based on dumbbell-shaped graphene metamaterial arrays. Phys. E 2018, 103, 93-98. [CrossRef]

12. Ren, J.; Wang, G.; Qiu, W.; Lin, Z.; Chen, H.; Qiu, P.; Wang, J.-X.; Kan, Q.; Pan, J.-Q. Optimization of the Fano Resonance Lineshape Based on Graphene Plasmonic Hexamer in Mid-Infrared Frequencies. Nanomaterials 2017, 7, 238. [CrossRef] [PubMed]

13. Liao, J.; Wu, X.; Liu, L.Y.; Xu, L. Fano resonance and improved sensing performance in a spectral-simplified optofluidic micro-bubble resonator by introducing selective modal losses. Opt. Express 2016, 24, 8574-8580. [CrossRef] [PubMed]

14. Wang, Y.F.; Liao, L.M.; Hu, T.; Luo, S.; Wu, L.; Wang, J.; Zhang, Z.; Xie, W.; Sun, L.X.; Kavokin, A.V.; et al. Exciton-Polariton Fano Resonance Driven by Second Harmonic Generation. Phys. Rev. Lett. 2017, 118, 063602. [CrossRef] [PubMed]

15. Lee, B.; Park, J.; Han, G.H.; Ee, H.S.; Naylor, C.H.; Liu, W.J.; Johnson, A.T.C.; Agarwal, R. Fano Resonance and Spectrally Modified Photoluminescence Enhancement in Monolayer $\mathrm{MoS}_{2}$ Integrated with Plasmonic Nanoantenna Array. Nano Lett. 2015, 15, 3646-3653. [CrossRef] [PubMed]

16. Black, L.J.; Wiecha, P.R.; Wang, Y.; de Groot, C.H.; Paillard, V.; Girard, C.; Arbouet, A. Tailoring Second-Harmonic Generation in Single L-Shaped Plasmonic Nanoantennas from the Capacitive to Conductive Coupling Regime. ACS Photonics 2015, 2, 1592-1601. [CrossRef]

17. Chen, J.; Xu, R.Q.; Mao, P.; Zhang, Y.T.; Liu, Y.J.; Tang, C.J.; Liu, J.Q. Realization of Fanolike resonance due to diffraction coupling of localized surface plasmon resonances in embedded nanoantenna arrays. Pasmonics 2015, 10, 341-346. [CrossRef]

18. Liu, Z.; Liu, G.; Fu, G.; Liu, X.; Wang, Y. Multi-band light perfect absorption by a metal layer-coupled dielectric metamaterial. Opt. Express 2016, 24, 5020-5025. [CrossRef] [PubMed]

19. Yi, Z.; Niu, G.; Ye, X.; Luo, J.S.; Li, X.B.; Jiang, X.D.; Huang, J.; Yi, Y.; Duan, T.; Zhang, J.C.; et al. Nanodisk-Induced Modification of Plasmon Coupling andAppearance of Fano Resonance Without Symmetry Breaking in Concentric Ag Nanoring-Nanodisk. Plasmonics 2017, 12, 889-898. [CrossRef] 
20. Rahmani, M.; Lei, D.Y.; Giannini, V.; Lukiyanchuk, B.; Ranjbar, M.; Liew, T.Y.F.; Hong, M.; Maier, S.A. Subgroup Decomposition of Plasmonic Resonances in Hybrid Oligomers: Modeling the Resonance Lineshape. Nano Lett. 2012, 12, 2101-2106. [CrossRef] [PubMed]

21. Zhan, Y.; Lei, D.Y.; Li, X.; Maier, S.A. Plasmonic FanonResonances in Nanohole Quadrumers for Ultra-Sensitive Refractive Index Sensing. Nanoscale 2014, 6, 4705-4715. [CrossRef] [PubMed]

22. Chen, Y.; Chu, J.R.; Xu, X.F. Plasmonic Multibowtie Aperture Antenna with Fano Resonance for Nanoscale Spectral Sorting. ACS Photonics 2016, 3, 1689-1697. [CrossRef]

23. Liu, Z.; Liu, X.; Huang, S.; Pan, P.; Chen, J.; Liu, G.; Gu, G. Automatically acquired broadband plasmonic-metamaterial black absorber during the metallic film-formation. ACS Appl. Mater. Interfaces 2015, 7, 4962-4968. [CrossRef] [PubMed]

24. Muhammad, N.; Khan, A.D.; Deng, Z.-L.; Khan, K.; Yadav, A.; Liu, Q.; Ouyang, Z. Plasmonic Spectral Splitting in Ring/Rod Metasurface. Nanomaterials 2017, 7, 397. [CrossRef] [PubMed]

25. Limonov, M.F.; Rybin, M.V.; Poddubny, A.N.; Kivshar, Y.S. Fano resonances in photonics. Nat. Photonics 2017, 11, 543-554. [CrossRef]

26. Chen, J.; Fan, W.F.; Zhang, T.; Tang, C.J.; Chen, X.Y.; Wu, J.J.; Li, D.Y.; Ying, Y. Engineering the magnetic plasmon resonances of metamaterials for high-quality sensing. Opt Express 2017, 25, 3675-3681. [CrossRef] [PubMed]

27. Cen, C.L.; Lin, H.; Liang, C.P.; Huang, J.; Chen, X.F.; Yi, Z.; Tang, Y.J.; Duan, T.; Xu, X.B.; Xiao, S.Y.; et al. Tunable plasmonic resonance absorption characteries-tics in periodic $\mathrm{H}$-shaped graphene arrays. Superlattices Microstruct. 2018, 120, 427-435. [CrossRef]

28. Yang, D.J.; Yang, Z.J.; Li, Y.Y.; Zhou, L.; Hao, Z.H.; Wang, Q.Q. Tunable Fano Resonance in Rod-Ring Plasmonic Nanocavities. Plasmonics 2015, 10, 263-269. [CrossRef]

29. Chen, Y.; Chen, Y.H.; Chu, J.; Xu, X.F. Bridged Bowtie Aperture Antenna for Producing an Electromagnetic Hot Spot. ACS Photonics 2017, 4, 567-575. [CrossRef]

30. Lin, J.Y.; Tsai, C.Y.; Lin, P.T.; Hsu, T.E.; Hsiao, C.F.; Lee, P.T. Optical Properties of Plasmonic Mirror-Image Nanoepsilon. Nanoscale Res. Lett. 2016, 11, 327. [CrossRef] [PubMed]

31. Johnson, P.B.; Christy, R.W. Optical constants of the noble metals. Phys. Rev. B 1972, 6, 4370. [CrossRef]

32. Palik, E.D. Handbook of Optical Constants of Solids; Academic Press: San Diego, CA, USA, 1985.

33. Koya, A.N.; Ji, B.; Hao, Z.; Lin, J. Resonance hybridization and near field properties of strongly coupled plasmonic ring dimer-rod nanosystem. J. Appl. Phys. 2015, 118, 113101. [CrossRef]

34. Aizpurua, J.; Hanarp, P.; Sutherland, D.S.; Kall, M.; Bryant, G.W.; Garcíade-Abajo, F.J. Optical properties of gold nanorings. Phys. Rev. Lett. 2003, 90, 057401. [CrossRef] [PubMed]

35. Link, S.; Mohamed, M.B.; El-Sayed, M.A. Simulation of the Optical Absorption Spectra of Gold Nanorods as a Function of Their Aspect Ratio and the Effect of the Medium Dielectric Constant. J. Phys. Chem. B 1999, 103, 3073-3077. [CrossRef]

36. Becker, J.; Andreas, T.; Jakab, A.; Hohenester, U.; Sönnichsen, C. The Optimal Aspect Ratio of Gold Nanorods for Plasmonic Bio-sensing. Plasmonics 2010, 5, 161-167. [CrossRef]

37. Yi, Z.; Niu, G.; Chen, J.F.; Luo, J.S.; Liu, X.N.; Yi, Y.; Duan, T.; Kang, X.L.; Ye, X.; Wu, P.H.; et al. Dipole, Quadrupole, and Octupole Plasmon Resonance Modes in Ag Nanoring Structure: Local Field Enhancement in the Visible and Near Infrared Regions. Plasmonics 2016, 11, 37-44. [CrossRef]

38. Xiao, S.Y.; Wang, T.; Liu, T.T.; Yan, X.C.; Li, Z.; Xu, C. Active modulation of electromagnetically induced transparency analogue in terahertz hybrid metal-graphene metamaterials. Carbon 2018, 126, 271-278. [CrossRef]

39. Chen, J.; Yi, Z.; Xiao, S.; Xu, X. Absorption enhancement in double-layer cross-shaped graphene arrays. Mater. Res. Express 2018, 5, 015605. [CrossRef]

40. Yang, Z.J.; Hao, Z.H.; Lin, H.Q.; Wang, Q.Q. Plasmonic Fano resonances in metallic nanorod complexes. Nanoscale 2014, 6, 4985-4997. [CrossRef] [PubMed]

(C) 2018 by the authors. Licensee MDPI, Basel, Switzerland. This article is an open access article distributed under the terms and conditions of the Creative Commons Attribution (CC BY) license (http:/ / creativecommons.org/licenses/by/4.0/). 